\title{
Hydroélectricité de forte puissance, autres usages de l'eau et reconquête de la biodiversité : une réflexion prospective sur les politiques publiques. Synthèse du séminaire du 4 novembre 2016
}

\author{
François GUERBER, Pierre-Alain ROCHE
}

Conseil général de l'environnement et du développement durable

\begin{abstract}
RÉSUMÉ. - Le séminaire ${ }^{1}$, organisé par le Conseil général de l'environnement et du développement durable sur les politiques publiques mettant en jeu de grands aménagements hydroélectriques, a rassemblé plus d'une centaine de participants d'origines très variées : représentants des divers types d'utilisation de l'eau (hydroélectricité, irrigation, biodiversité, protection contre les inondations, pêche, navigation, loisirs nautiques), gestionnaires de la ressource (établissement public territorial de bassin, agences de l'eau), bureaux d'études et administrations.

Bien que moins médiatisés ces derniers temps que les débats sur les moulins et la «petite » hydraulique, les enjeux de la production hydroélectrique de forte puissance sont majeurs en raison de sa place exceptionnelle dans le mix énergétique, de l'impact des ouvrages sur la biodiversité et des multiples autres usages auxquels les grandes retenues peuvent contribuer. Les possibilités d'évolution sont importantes mais tout autant les difficultés à concilier les objectifs sectoriels qui parfois s'opposent. Les échanges ont mis en lumière les tensions entre les acteurs mais aussi des cas concrets et les facteurs clés d'une gestion intégrée réussie ; les échanges ont souligné la complexité des politiques publiques mais ont aussi identifié les outils ou avancées méthodologiques utilisables, parfois peu connues des décideurs. Au final, la recherche de solutions semble passer par l'explicitation partagée de cette complexité plutôt que par des combats médiatiques trop simplificateurs.
\end{abstract}

Mots-clés : énergie hydraulique, environnement, impact, aménagement hydraulique

\section{High-power hydroelectricity, other uses of water and reconquest of biodiversity: a prospective thinking on public policies. Synthesis of the seminar of 4 November 2016}

\begin{abstract}
The seminar, organized by the General Council on Environment and Sustainable Development about public policies involving large hydroelectric installations, brought together over a hundred participants from a wide range of backgrounds: representatives of various types of water users (hydroelectricity, irrigation, biodiversity, flood protection, fishing, navigation, water recreation), water resources managers (public territorial watershed organization, water agencies), consultants and administrations.

Although less publicized lately than debates on mills and "small" hydraulics, the stakes of high-power hydro-electric production are major because of its exceptional place in the energy mix, because of the impact of the infrastructures on biodiversity and because of the many other uses to which large reservoirs can contribute. The possibilities for change are important, but also the difficulties in reconciling the sectoral objectives that are sometimes opposed. The discussions highlighted the tensions between the actors but also concrete cases and key success factors for integrated management ; the discussions underlined the complexity of public policies but also identified the usable tools or methodological advances, which are sometimes little known to decision makers. In the end, the search for solutions seems to go through the shared explanation of this complexity rather than through too simplistic media fights.
\end{abstract}

Key-words: hydraulic power, environnement, impact, Hydraulic infrastructure

\section{UNE PROBLÉMATIQUE PARTICULIÈREMENT D'ACTUALITÉ}

Comment les nombreux « usages $»^{2}$ de la ressource en eau peuvent-ils cohabiter et évoluer à l'avenir autour d'une même rivière lorsque celle-ci est déjà équipée de

1. Référence en fin d'article

2. On entend par là les différentes activités qui prélèvent de l'eau dans les rivières, telles que l'alimentation en eau potable, l'irrigation, l'utilisation par l'industrie pour process, refroidissement ou lavage, la production d'énergie par turbinage, l'alimentation des canaux de navigation, les activités grands aménagements de production hydroélectrique concédés?

La problématique est très actuelle et se décline en plusieurs questions :

- de nombreux projets visent à satisfaire de nouveaux besoins en exploitant des volumes d'eau stockés dans les réserves des 


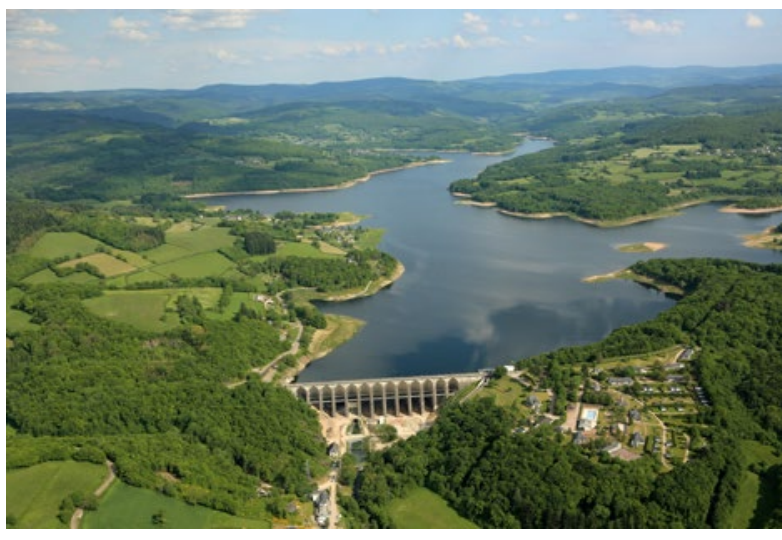

Fig. 1 : barrage de Pannecière Chaumard (Seine grands lacs)

barrages hydroélectriques : comment trouver les équilibres, valoriser les pertes de productions et les financer?

- la loi pour la reconquête de la biodiversité adoptée en juillet 2016 fixe de nouveaux principes et procédures non seulement pour équiper les ouvrages mais aussi pour les gérer différemment : quelles conséquences concrètes faut-il en attendre ?

- la programmation pluriannuelle de l'électricité adoptée par décret fin octobre 2016 précise les objectifs et orientations pour les années à venir qui permettront de respecter les accords de Paris en matière de climat : comment cette programmation générale mais sectorielle sera-t-elle rendue cohérente avec les autres contraintes ou besoins locaux ?

- enfin le renouvellement de nombreuses concessions hydroélectriques est en préparation pour les années à venir selon des procédures qui ont été précisées récemment par la réglementation : comment les usages de l'eau autres que la production d'énergie, souvent installés depuis de nombreuses années et très en aval, seront-ils pris en compte dans ces procédures conduites à une échelle territoriale réduite ?

Lorsqu'ils sont évoqués à l'échelle nationale, les enjeux et politiques sectoriels apparaissent divergents et les parties prenantes semblent être en conflit

Les grands aménagements hydrauliques jouent un rôle majeur dans la production d'énergie électrique en France : ils sont à l'origine de la grande majorité de l'hydroélectricité qui représente $12 \%$ du mix électrique et constitue la première source d'électricité renouvelable (Fig. 2)

L'intérêt énergétique exceptionnel de ces aménagements tient à leur flexibilité : ils peuvent monter en production très rapidement, et répondre ainsi à trois objectifs essentiels :

- produire une énergie renouvelable ;

- couvrir les besoins énergétiques de pointe, l'énergie produite étant ainsi vendue au meilleur prix ;

- satisfaire, pour plus de la moitié, le besoin croissant d'ajustement ${ }^{3}$, qui compense la forte variabilité de la production d'électricité d'origine solaire ou éolienne.

Pour les producteurs ${ }^{4}$ d'énergie d'origine hydraulique, les recettes se sont réduites depuis quelques années pour deux raisons :

- la vente de l'énergie, réalisée autrefois selon un tarif réglementé, est aujourd'hui ouverte à la concurrence et le prix librement établi se trouve actuellement trois fois plus bas que les tarifs antérieurs ;

3. Il s'agit du dispositif par lequel RTE adapte les moyens de production à la consommation réellement appelée.

4. Dans cet article, sauf mention explicite contraire, les producteurs dont il s'agit sont ceux qui exploitent des ouvrages de forte puissance.
- la quantité d'énergie productible étant dans le même temps orientée à la baisse sous la pression d'autres usages, dont la biodiversité, l'équilibre économique des aménagements est de plus en plus difficile à assurer ou même devient hors de portée.

Les protecteurs de la nature mettent en avant les impacts environnementaux de la production hydroélectrique : augmentation de température, eutrophisation, transport solide, faune aquatique. Ils ne remettent pas en cause les grands aménagements, notamment en raison de leurs fonctions multiples. Mais ils demandent que les augmentations de production programmées en France ne se traduisent pas par la création d'un très grand nombre d'installations de petite taille, ce qui ne prendrait pas suffisamment en compte les objectifs de continuité écologique ou de biodiversité. Ils souhaitent que le choix des sites à équiper soit effectué dans la plus grande transparence et selon le principe de la séquence " éviter, réduire, compenser ».

Pour les irrigants, il est essentiel de maintenir l'agriculture des régions méditerranéennes, qui est particulièrement menacée par l'urbanisation, et par le changement climatique qui renforce la demande en eau et diminue la ressource. Il faut mettre en place les ouvrages complémentaires et les protections réglementaires qui permettent au moins d'enrayer la disparition (jusqu'à $5 \%$ par an) de systèmes d'irrigation existants.

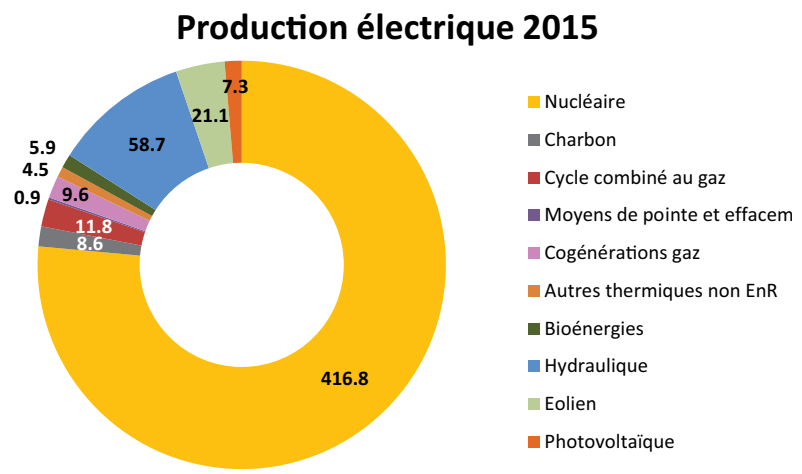

\section{Répartition de la production d'électricité d'origine renouvelable}

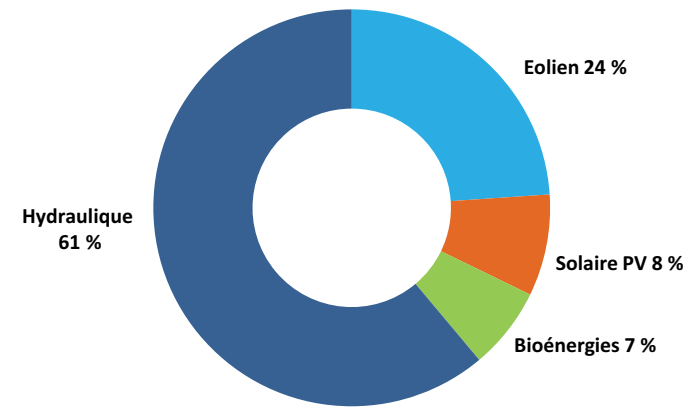

Fig. 2 : Répartition de la production d'énergie

A l'échelle locale pourtant, des acteurs ont su trouver un équilibre dans l'utilisation de la ressource en eau en fonction des aménagements existants. Quatre exemples illustrent ces réussites.

La Compagnie d'aménagement des Coteaux de Gascogne, au travers du « système Neste » qui relie plusieurs affluents de rive gauche de la Garonne influencés par de grands aménagements hydroélectriques, satisfait six usages de l'eau différents. La concertation régulière permet de s'accorder sur la répartition de l'eau, malgré des différences considérables 


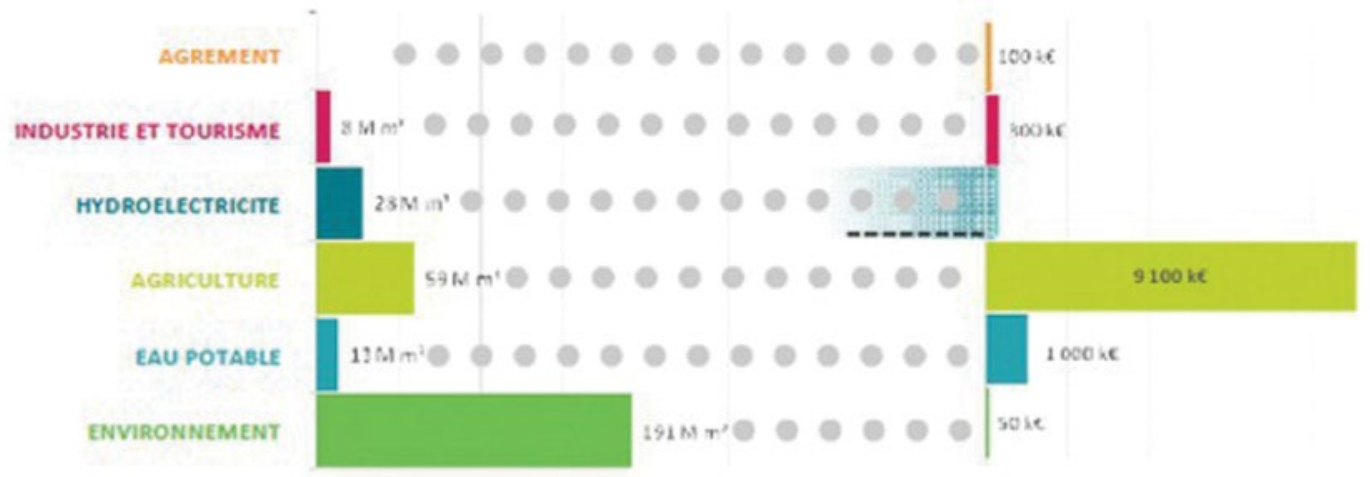

Fig. 3 : Comparaison volume consommé et coût

entre les volumes demandés et les retombées économiques pour chaque usage, comme le présente la figure 3.

L'Entente interdépartementale du bassin du Lot a mis en place, entre 1989 et 1994, un accord " gagnant - gagnant » entre EDF, le département et les usagers : une contribution financière, prévue par le conseil départemental pour un barrage qui finalement n'a pas été construit, a été convertie en un achat forfaitisé d'un débit de soutien d'étiage de $10 \mathrm{~m}^{3} / \mathrm{s}$, jusqu'à la fin de la concession d'un ouvrage préexistant. Les usages ont donc pu se développer comme l'illustre la Figure 4, par le biais d'une concertation régulière des acteurs à l'échelle de l'ensemble du bassin versant. Aujourd'hui, l'établissement public s'inquiète pour la survie de ces activités, car il ne sait pas si ce soutien d'étiage sera repris comme une contrainte à respecter dans le cahier des charges de la future concession. En effet, les textes récents concernant le renouvellement des concessions prévoient une concertation et des recettes à l'échelle d'un territoire beaucoup plus réduit (groupement de communes où sont situés les ouvrages) que le bassin influencé par les ouvrages.

Pour gérer les barrages réservoirs du bassin de la Seine, représentant $800 \mathrm{Mm}^{3}$ et construits jusqu'aux années 80 , l'établissement public Seine-Grands lacs a élargi les objectifs initiaux - de protection de l'agglomération contre les crues et de soutien des étiages - au tourisme, à la biodiversité et la production d'électricité. Des études conduites récemment indiquent comment ré-optimiser les consignes de gestion pour les adapter au changement climatique. La gouvernance aussi devra évoluer pour prendre en compte les besoins de territoires situés entre les barrages et l'agglomération parisienne.

\section{GARANTIR LE MULTI-USAGE DE L'EAU EN PRÉSERVANT LE PRINCIPE DE SOLIDARITÉ AMONT-AVAL}

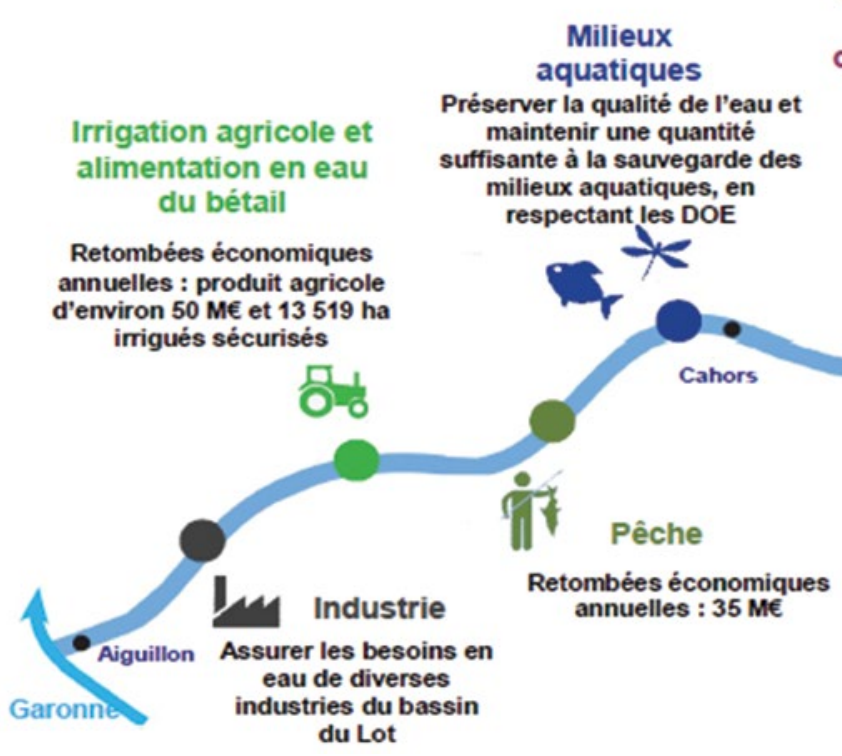

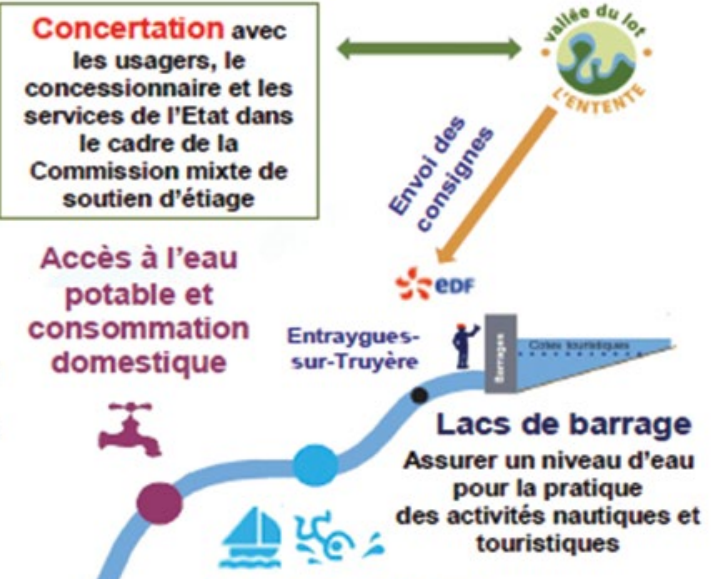

Activités nautiques

Retombées économiques annuelles : $15 \mathrm{M \epsilon}$

Fig. 4 : Multi-usage de l'eau, exemple de la vallée du Lot 
La Compagnie nationale du Rhône produit 25 \% de l'énergie hydroélectrique française avec une puissance de 3 TWh. L'aménagement du Rhône comprend 23 barrages, une quarantaine d'écluses pour le transport fluvial et la navigation de plaisance, et des prises d'eau pour l'irrigation de 120000 ha (Fig. 5). Les besoins environnementaux sont pris en compte au travers des « missions d'intérêt général » qui figurent au cahier des charges de la concession, et qui font l'objet de programmations pluriannuelles négociées avec l'État et bénéficiant de l'appui de l'agence de l'eau. Sont ainsi développés des programmes de remise en eau et de restauration des habitats dans les tronçons de cours d'eau court-circuités, ou des ouvrages de circulation pour les poissons migrateurs, avec pour objectif d'atteindre $100 \%$ de masses d'eau en bon état en 2027.

Un autre cas, dont le redémarrage a été annoncé peu après le séminaire, est le projet d'hydroélectricité durable dans les gorges de l'Allier, "vers le nouveau Poutès ", qui est reconnu comme exemplaire par tous les acteurs. Ayant évolué d'une situation de conflit il y a 20 ans à une gouvernance partagée, les parties prenantes ont construit ensemble un projet qui à la fois maintiendra une grande partie $(85 \%)$ de la production d'énergie renouvelable sur l'ouvrage actuel et restaurera en bonne partie le fonctionnement hydrologique et la biodiversité du bassin.

\section{Aménagements CNR}

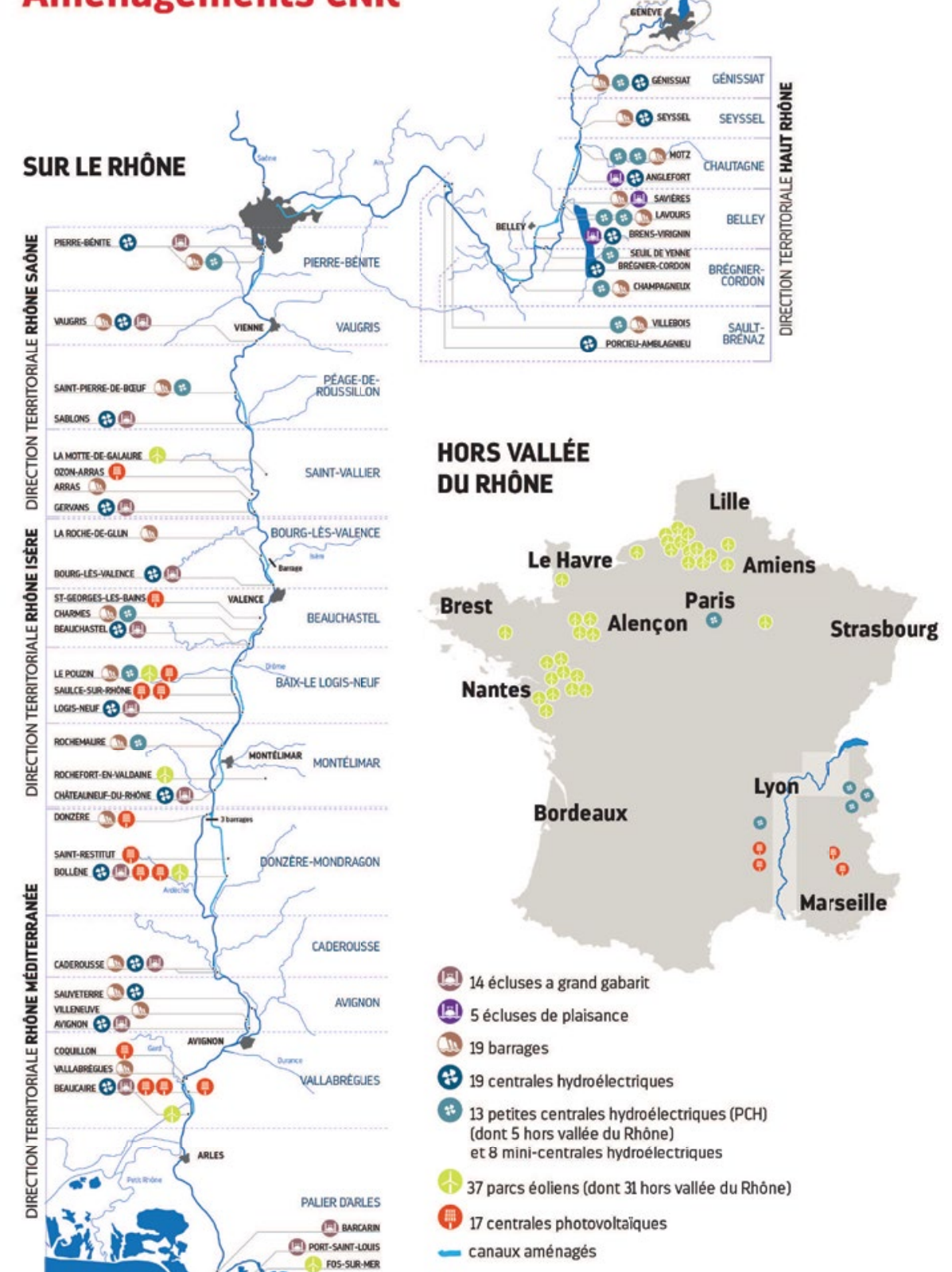

\section{A L'ÉTRANGER}

\& 2 petites centrales hydroélectriques à Dardha (Albanie) 
Les outils et méthodes de la "gestion intégrée des ressources en eau " sont-ils efficaces pour pour rendre plus cohérentes les politiques publiques ?

La concertation multi-acteurs est toujours citée comme la clé des expériences réussies. Les premiers "outils " à utiliser sont donc les méthodes de concertation. L'analyse des concertations mises en œuvre dans les schémas d'aménagement et de gestion des eaux (SAGE) fait ressortir les conditions de réussite suivantes :

- une bonne représentativité des acteurs réunis au sein de la Commission locale de l'eau (CLE) qui doit articuler les problématiques locales avec les objectifs généraux du schéma directeur d'aménagement et de gestion des eaux (SDAGE) ; - une volonté collective d'assurer la viabilité économique et environnementale de la gestion des ouvrages communs;

- des limites du territoire bien choisies au regard des actions à mener ;

- la disponibilité d'informations de qualité.

Ces conditions ne sont pas toujours réunies, et il est rare de partager complètement des enjeux aussi divers et contradictoires que ceux de la transition énergétique, de l'agriculture et de la biodiversité, en période de changement climatique.

Les innovations technologiques telles que les turbines ichtyocompatibles (qui réduisent fortement les mortalités des poissons lors de leur passage) ou les prises d'eau « Coanda » (qui évitent l'entrée des débris et des poissons dans les turbines) et les outils techniques, tels que les passes à poissons, sont rassemblés dans le guide "RefMADI Hydroélec $»^{5}$. Les concepteurs de projet en disposent pour homogénéiser leurs études d'impact et réduire les impacts sur la biodiversité. Il faut néanmoins garder à l'esprit que biodiversité n'est pas synonyme de renforcement du débit d'étiage et que, si des améliorations existent pour la continuité écologique et le transport des sédiments, il n'y a pas de moyen de réduire l'impact dû à l'ennoiement des zones situées en amont des ouvrages. Le partage des enjeux très en anticipation des projets ainsi que la prise en compte des effets cumulés sur tout le bassin versant sont nécessaires pour lancer les concertations sur de bonnes bases techniques.

La gestion des aménagements hydroélectriques en temps réel a progressé par le développement des outils d'optimisation en avenir incertain fondés sur des techniques de scénarios. Ces modélisations stochastiques intègrent des données hydrologiques (prévisions d'apports), techniques (caractéristiques des ouvrages et de chaînes d'ouvrages) et économiques (prévisions de la valeur instantanée, très fluctuante, de la production sur le marché européen de l'énergie). Les usages autres que la production d'électricité sont généralement pris comme des contraintes à respecter et on ne peut donc pas véritablement parler d'optimisation multi-objectifs. Des démarches d'optimisation conjointe de plusieurs types d'usages de l'eau restent à développer, utilisant des théories plus diversifiées de valorisation économique.

L'optimisation technico-économique d'un ensemble d'usages diversifiés a parfois été appliquée en phase de planification d'un ensemble d'investissements (barrages et centrales hydroélectriques mais aussi périmètres d'irrigation, endiguements, traitement de l'eau, installations portuaires, etc.): lorsque les États riverains du fleuve Sénégal ont construit les barrages à buts multiples du bassin versant, grâce à la simulation de scénarios technico-économiques multi-sectoriels, ils se sont accordés sur les répartitions par

5. Référentiel milieux aquatiques. Documents d'incidence appliqués à l'hydroélectricité.

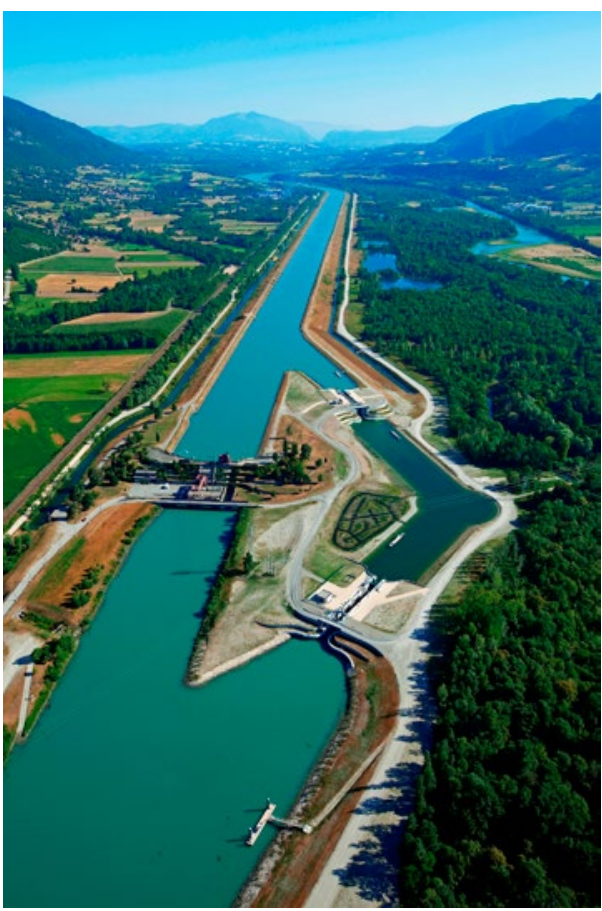

Fig. 6 : Aménagement de Chautagne, Anglefort(AIN 01) photo Camille MOIRENC, photothèque CNR

pays et par secteur des utilisations des grandes quantités d'eau mobilisées ainsi que des remboursements des prêts accordés par les organismes de financement internationaux.

Enfin, l'utilisation d'outils socio-politiques est à développer, car il est irréaliste de croire que la concertation sera toujours de type « gagnant - gagnant ». Certaines concertations seront conflictuelles et il existe des méthodes pour anticiper et gérer ce type de situation. Certaines conciliations pourront évoluer selon le cadre qui sera fixé aux acteurs : par exemple si on passe d'un cadrage qui vise le retour du saumon dans l'Allier à un cadrage qui vise le retour du saumon en France, cela modifiera les possibilités de compensation et les relations entre les acteurs. Enfin, certains usagers seront « perdants » dans la mesure où ils devront réduire leur consommation d'eau à l'issue des négociations. Mais les solutions ne viendront pas uniquement de la gestion de l'eau : les sciences sociales ont à identifier des évolutions plus larges des modes de vie, qui peuvent se révéler plus durables car moins consommatrices d'eau ou d'énergie, telles que la réduction de la consommation de viande ou la diminution des déplacements physiques. Les acteurs locaux pourront ainsi mieux développer les politiques les plus résilientes aux changements à venir.

\section{REMERCIEMENTS}

François Guerber a pu écrire cet article avec Pierre-Alain Roche grâce aux tables rondes du séminaire qu'ils ont animées avec François Cholley, CGE, et qui ont fait intervenir : Thibaud Normand, chef du bureau de la production électrique, DGEC ; Yves Giraud, directeur de la production hydraulique d'ÉdF ; Jacques Pulou, Fédération Rhône-Alpes de Protection de la Nature ; Michel Pontier, Président de l'Association des irrigants de la région méditerranéenne ; Alain Poncet, DG de la Compagnie d'aménagement des coteaux de Gascogne ; Serge Bladinières et Marie-Hélène Privat, Président et directrice de l'Entente interdépartementale du bassin du Lot ; Régis Thépot, DG de Seine grands lacs ; Eric Divet, Directeur 
$\mathrm{du}$ patrimoine fluvial et industriel, Compagnie nationale $\mathrm{du}$ Rhône ; Yves Picoche, Directeur des interventions et actions de bassin, Agence de l'eau RM\&C ; Maxime Tirman, responsable du groupement d'usines de Serre-Ponçon, EdF ; Dominique Baril, Directeur de la cellule «Ingénierie écologique», ONEMA ; Antoine Langumier, DG de Ecodécision ; Gabrielle Bouleau, chercheuse en science politique, IRSTEA.

\section{RÉFÉRENCE}

CGedd (2016) - . Actes du Séminaire Hydroélectricité, autres usages et reconquête de la Biodiversité, 4 Novembre 2016, Téléchargeable sur http://www.cgedd.developpement-durable. gouv.fr/seminaire-hydroelectricite-autres-usages-et-a2320.html

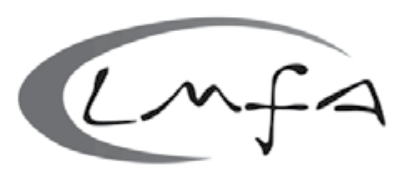

\section{LABORATOIRE DE MECANIQUE DES FLUIDES ET D'ACOUSTIQUE UMR CNRS 5509}

Le LMFA est une Unité Mixte de Recherche (UMR 5509) entre le CNRS, l'Ecole Centrale de Lyon, I'Université Claude Bernard Lyon 1 et l'INSA de Lyon.

L'Unité regroupe 113 permanents et 86 doctorants et postdoctorants, qui conduisent des recherches fondamentales et finalisées dans quatre grands domaines : l'Acoustique, la Turbulence et les Instabilités, les Turbomachines et les Fluides complexes et Transferts.

Une part notable des travaux concerne l'Environnement, dans sa composante atmosphérique comme sa composante hydraulique (écoulements polyphasiques, transports sédimentaires, inondations...).

Le LMFA dispose de plusieurs gros équipements expérimentaux (souffleries subsonique et supersonique associées à une chambre anéchoïque, soufflerie atmosphérique, bancs Turbomachines, canaux hydrauliques), ainsi que d'outils de simulation numérique performants.

Le LMFA développe de nombreux partenariats avec les entreprises ayant des besoins de recherche avancée en Mécanique des Fluides et en Acoustique.

\section{Contact philippe.blanc-benon@ec-lyon.fr} www.Imfa.ec-lyon.fr 